\title{
Geometric Frustration of Colloidal Dimers on a Honeycomb Magnetic Lattice
}

\author{
Pietro Tierno* \\ Estructura i Constituents de la Matèria, Universitat de Barcelona, 08028 Barcelona, Spain \\ and Institut de Nanociència i Nanotecnologia, Universitat de Barcelona, 08028 Barcelona, Spain \\ (Received 25 August 2015; revised manuscript received 19 October 2015; published 22 January 2016)

\begin{abstract}
We study the phase behavior and the collective dynamics of interacting paramagnetic colloids assembled above a honeycomb lattice of triangular shaped magnetic minima. A frustrated colloidal molecular crystal is realized when filling these potential minima with exactly two particles per pinning site. External in-plane rotating fields are used to anneal the system into different phases, including long range ordered stripes, random fully packed loops, labyrinth and disordered states. At a higher amplitude of the annealing field, the dimer lattice displays a two-step melting transition where the initially immobile dimers perform first localized rotations and later break up by exchanging particles across consecutive lattice minima.
\end{abstract}

DOI: 10.1103/PhysRevLett.116.038303

Geometric frustration arises when the spatial arrangement of the system elements prevents simultaneous minimization of all interaction energies, and features at low temperature a highly degenerate ground state [1]. Effects of such phenomenon manifest in disparate systems, from classical magnets [2,3] to active matter [4], coupled lasers [5], complex networks [6], and quantum many-body systems [7-9]. Recent experiments with size-tuneable microgel particles [10] have shown that strongly confined colloids represent a versatile model to investigate geometrically frustrated states. In contrast to lattices of interacting nanoscale islands such as artificial spin ice [11,12], colloids feature time and length scales that are accessible via simple light microscopy, combined with the possibility to control in situ the pair interaction via external fields.

Above a periodic potential, microscopic particles can be arranged into colloidal molecular crystals [13], i.e., lattices of doublets, triplets, or larger clusters characterized by internal rotational degrees of freedom [14]. While colloidal molecular crystals are excellent models to study geometric frustration effects due to competing orientational order and lattice constraints [15-18], the focus of these experiments has been placed mainly on the melting scenario of trimer systems on a triangular lattice [13]. On this lattice, trimers can be arranged only in one of two orientational states, while dimers present a richer phase behavior due to the larger number of possible configurations between pairs [17]. The lattice covering by dimer particles is also a fascinating problem in statistical mechanics [19], which has been recently the subject of renewed theoretical interest $[20,21]$, in addition to being present in different processes like melting [18], self-assembly [22], and molecular adsorption on a crystalline surface [23].

This Letter investigates the colloidal ordering and dynamics of interacting microscopic dimers self-assembled above a honeycomb magnetic lattice. Each dimer is composed of a pair of paramagnetic colloids confined in a triangular shaped magnetic minimum. An external precessing field sets the dimers into rotational motion, annealing the lattice to a minimum energy state. Depending on the field parameters, the resulting dimer arrangement can be mapped to a long range striped phase or to a random fully packed loop (FPL) state. On a honeycomb lattice a FPL configuration can be constructed by considering a series of arrows that joins the lattice vertices. Each vertex generates one arrow, which ends at one of the three nearest vertices. All arrows have the same length and form a series of closed and self-avoiding loops. These loops are not allowed to have free ends, a condition that strongly limits the number of ways the arrows can be placed. FPL models have been used to explain a broad class of phenomena in magnetism, optics, and polymer physics [24-28], but physical realizations are rather scarce. Dimers on a hexagonal lattice can be effectively arranged in such a way to produce a FPL configuration [29]. More recently, FPLs have been predicted to appear for isotropically interacting colloids arranged above a honeycomb lattice of triangular shaped optical traps [30]. Here, this idealized state is experimentally reported using magnetic dimers interacting via dipolar forces and arranged above a magnetic lattice.

The dimers are composed of pairs of monodisperse paramagnetic colloids having a diameter $d=2.8 \mu \mathrm{m}$ and a magnetic volume susceptibility $\chi \sim 0.4$ (Dynabeads M-270, Dynal). Because of the doping with superparamagnetic iron oxide grains, these particles acquire a dipole moment $\boldsymbol{m}=\left(\pi d^{3} / 6\right) \chi \boldsymbol{H}$, when subjected to an external field $\boldsymbol{H}$. The particles are dispersed in deionized water and deposited above a uniaxial ferrite garnet film (FGF) having thickness $t \sim 4 \mu \mathrm{m}$ and saturation magnetization $M_{s}=$ $1.7 \times 10^{4} \mathrm{~A} \mathrm{~m}^{-1}$ [31]. The FGF displays a triangular lattice of magnetic "bubbles," i.e., cylindrical ferromagnetic domains uniformly magnetized and immersed in an opposite magnetized film, Fig. 1(a). The size of the bubble domains can be easily controlled by a magnetic field 


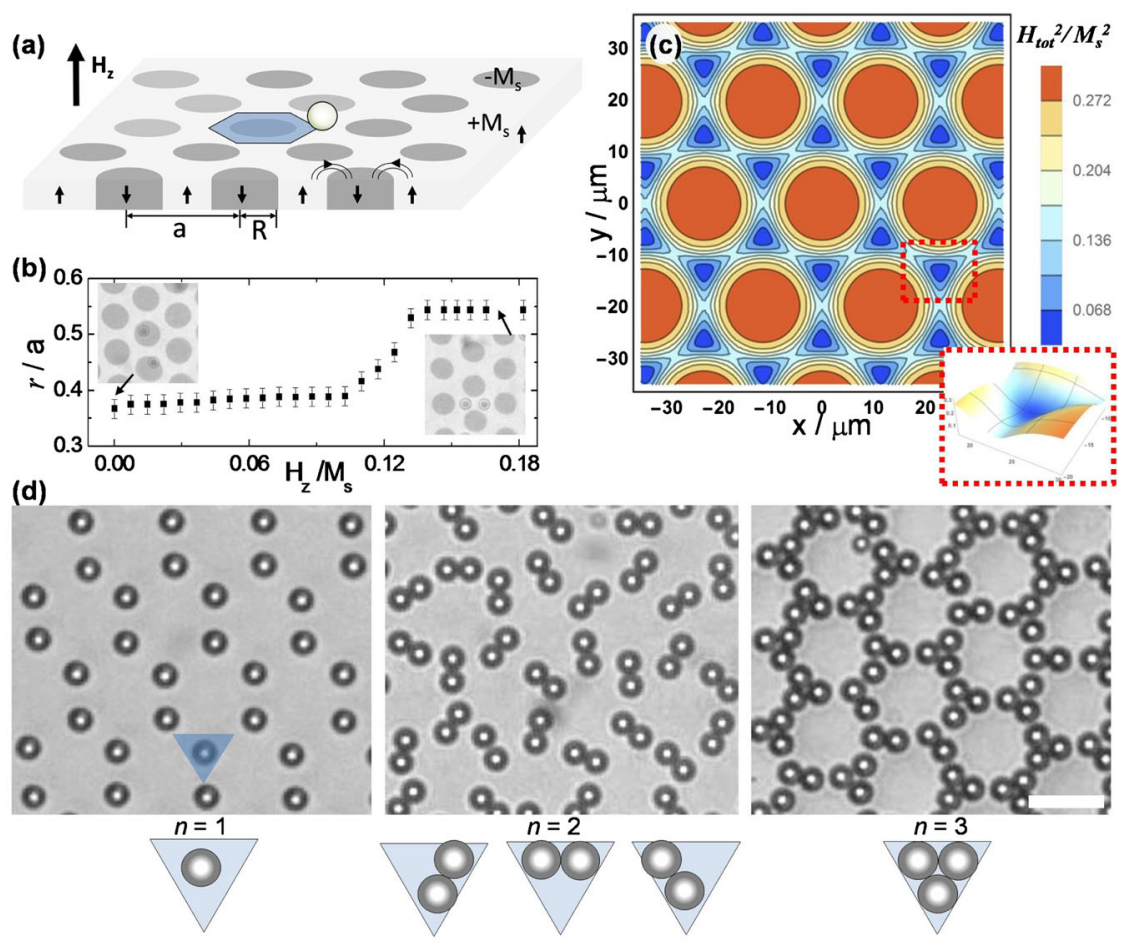

FIG. 1. (a) Schematic of the FGF film with a magnetic bubble lattice subjected to an external field $\boldsymbol{H}_{z}$. One Wigner-Seitz unit cell is shaded in blue with one paramagnetic colloid. For $H_{z}=0$, the lattice constant is $a=11.6 \mu \mathrm{m}$ and the radius $R=4.2 \mu \mathrm{m}$. (b) Normalized distance $r / a$ of one paramagnetic colloid from the center of a magnetic bubble versus $H_{z}$. (c) Normalized energy landscape for a FGF under a static field $H_{z}=0.17 M_{s}$. The inset shows a 3D view of one triangular minimum. (d) Snapshots of a small section $\left(48 \times 42 \mu \mathrm{m}^{2}\right)$ of a magnetic bubble lattice filled with $n=1,2,3$ particles per pinning site (scale bar is $10 \mu \mathrm{m}$ ). Schematics at the bottom show the corresponding configurations in a triangular minimum. applied perpendicular to the FGF, $\boldsymbol{H}_{z}=H_{z} \boldsymbol{e}_{z}$, and for amplitudes $H_{z} \lesssim 0.3 M_{s}$ the bubble radius varies linearly with $H_{z}$ (Fig. S1 in Ref. [31]). Once above the FGF surface, the particles pin at the Bloch walls, which are located at the perimeter of the magnetic bubbles. However, for a perpendicular field $H_{z}>0.14 M_{s}$, the equilibrium position of the colloids is shifted in the interstitial region, i.e., at the vertices of the Wigner-Seitz unit cell around the bubbles, Fig. 1(b). Under these conditions, the location of the magnetic minima can be visualized by calculating the energy of a paramagnetic colloid $U_{m} \sim H_{\text {tot }}^{2}$ subjected to the global field $\boldsymbol{H}_{\text {tot }}=\boldsymbol{H}+\boldsymbol{H}_{\text {stray }}, \boldsymbol{H}$ being the applied field and $\boldsymbol{H}_{\text {stray }}$ the stray field of the FGF [31]. As shown in Fig. 1(c), the energy landscape displays a honeycomb lattice of triangular shaped minima having alternating orientations. From the small inset in Fig. 1(c) (see also Fig. S5 in Ref. [31]) it follows that these minima have one deep central well and three higher wells at the edges of the triangle. This feature explains the particle location at different filling ratios, as shown in Fig. 1(d). With one particle per pinning site the colloids replicate the honeycomb lattice of magnetic minima. Dimers are formed with two particles, and have three energetically equivalent states, since the excluded volume between these particles impedes occupation of the central well. In contrast, for trimers this energetic degeneracy disappears since each particle can sit close to one of the three corners of the triangle. Here, we focus on the dimer system, which is characterized by frustrated interactions and a degenerate ground state.

Before each experiment, we prepare an initial disordered configuration of dimers having a random distribution of the three orientations. The annealing procedures used to generate this configuration and later to order the system into different phases are both obtained by superimposing on $\boldsymbol{H}_{z}$ a rotating in-plane field $\boldsymbol{H}_{x y}=H_{x y}\left[\cos (\omega t) \boldsymbol{e}_{x}+\sin (\omega t) \boldsymbol{e}_{y}\right]$. The resulting field $\boldsymbol{H}=\boldsymbol{H}_{x y}+\boldsymbol{H}_{z}$ performs a conical precession around the $z$ axis with angular frequency $\omega$. In the preannealing process, we use higher amplitudes $\left(H_{z}=0.25 M_{s}\right.$, $\left.H_{x y}=0.20 M_{s}\right)$ such that the modulated landscape forces the exchange of particles between nearest interstitials and consequently randomizes the dimer orientations. Afterwards, the static field is decreased to $H_{z}=0.17 M_{s}$ and $\boldsymbol{H}_{x y}$ is reduced to zero at a rate of $0.02 M_{s} s^{-1}$. Once prepared, the lattice of dimers with disordered orientations is rather stable with negligible spontaneous rotations of the dimers due to thermal fluctuations [43].

In order to anneal the lattice into an ordered phase, a rotating field with amplitude $H_{x y} \in[0.06,0.14] M_{s}$ is used, such that it forces the rotational motion of the dimers but does not produce an exchange of particles between consecutive interstitial regions. Figure 2(a) and MovieS1 in Ref. [31] show the effect of a rotating field with $H_{x y}=$ $0.07 M_{s}$ on the dimer orientation. The bottom row in Fig. 2(a) illustrates how the energy landscape is altered by the inplane field during one cycle. The applied field modifies the stray field of the FGF deforming the triangular minima such that they can accommodate now a dimer only in one orientation. The resulting phase is a long range nematic order characterized by dimers with alternating orientations. Figure 2(b) shows the average rotational speed $\langle\dot{\varphi}\rangle$ as a function of $\omega$ for a single $(s)$ dimer and for a lattice $(l)$ of dimers due to this annealing field. The dynamics for an isolated dimer can be well described as a standard desynchronization process in a dissipative medium: below a critical 
frequency $\omega_{c}^{s}=73.2 \mathrm{rad} \mathrm{s}^{-1}$ the dimer follows the rotating field with a constant phase-lag angle, while for $\omega>\omega_{c}^{s}$ there is an asynchronous regime where $\langle\dot{\varphi}\rangle$ decreases as $\omega$ increases. Neglecting thermal fluctuations, one can fit well the experimental data using the solution of the Adler equation [45] $\langle\dot{\varphi}\rangle / \omega=1-\left[1-\left(\omega_{c}^{s} / \omega\right)^{2}\right]^{(1 / 2)}$. At parity of the field parameters the lattice of dimers desynchronizes earlier, with a critical frequency $\omega_{c}^{l}=49.9 \mathrm{rad} \mathrm{s}^{-1}<\omega_{c}^{s}$, and displays a faster decay of $\langle\dot{\varphi}\rangle$ in the asynchronous regime. When increasing $H_{x y}$ the particle induced moment also increases since $\boldsymbol{m} \sim\left(\boldsymbol{H}+\boldsymbol{H}_{\text {stray }}\right)$, and thus dipolar interactions between close dimers become important, competing with the orientation imposed by the substrate. Indeed, we find that the separation frequency $\Delta \omega=\omega_{c}^{s}-\omega_{c}^{l}$ between the two critical frequencies increases with rising $H_{x y}$, Fig. S2 in Ref. [31]. As shown in the small inset in Fig. 2(b), the induced moments in the particles are mainly oriented in the plane of the FGF due to the configuration of (a)

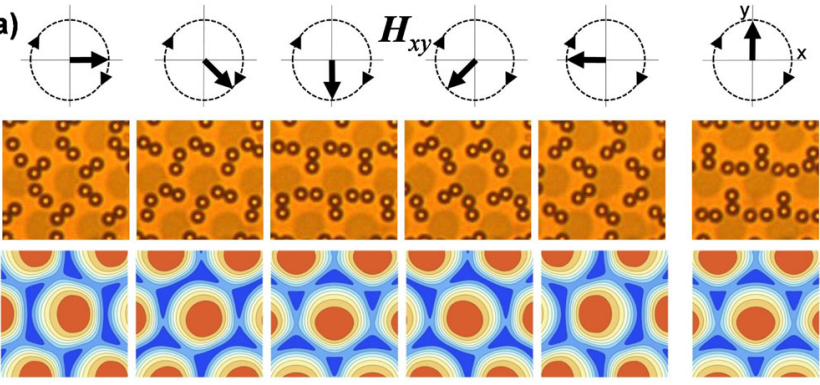

(b)

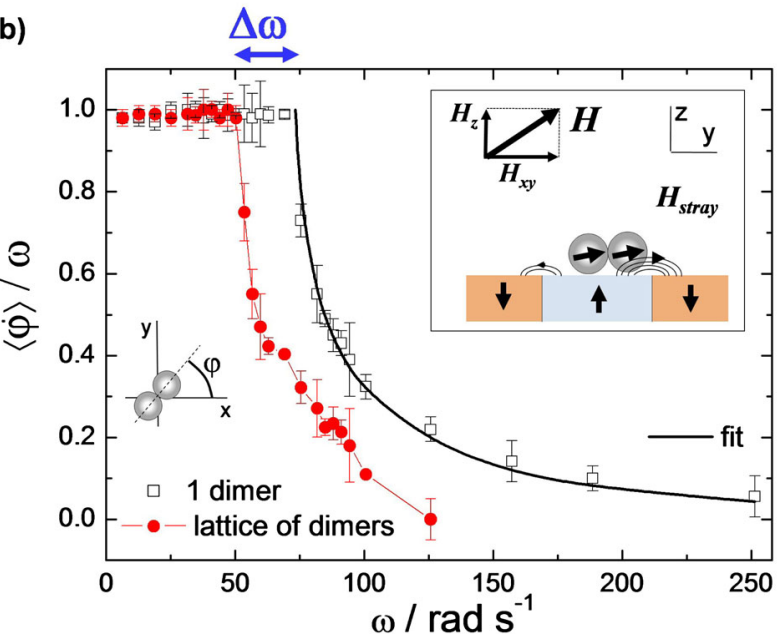

FIG. 2. (a) Polarization microscopy images showing the locations of the dimers and of the magnetic bubbles with directions of the annealing field $\left(H_{x y}=0.07 M_{s}, \omega=6.3 \mathrm{rad} \mathrm{s}^{-1}\right.$, MovieS1 [31]). Images at the bottom show the corresponding energy landscape deformed by the field; maxima are colored in red, minima in blue. (b) Average rotational speed $\langle\dot{\varphi}\rangle$ versus angular frequency $\omega$ for a single dimer (empty squares) and for a lattice of interacting dimers (filled circles) $\left(H_{x y}=0.08 M_{s}\right)$. From the fit (equation in the text) the critical frequency is $\omega_{c}^{s}=73.2 \mathrm{rad} \mathrm{s}^{-1}$. Inset: schematic showing the side view of a dimer above the FGF. (a)

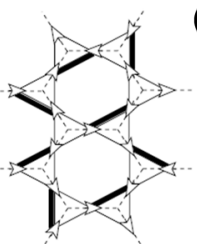

(b)

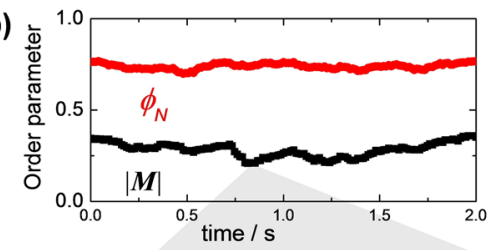

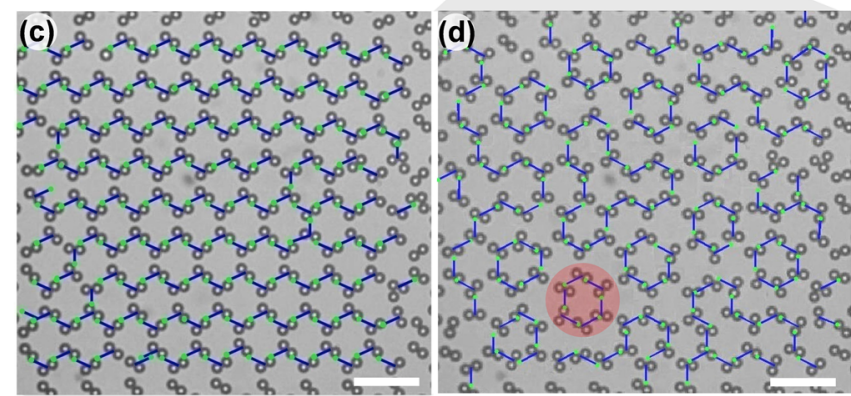

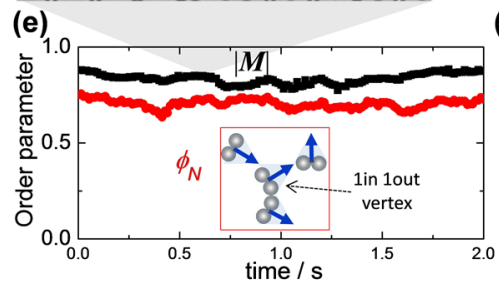

(f)

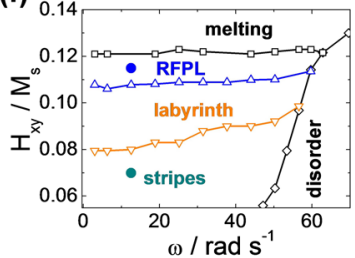

FIG. 3. (a) Arrow representation of dimers covering a kagome lattice; the image is reproduced from Ref. [32]. (b) Order parameters $|\boldsymbol{M}|$ and $\phi_{N}$ versus time for (d). (c) Stripe phase of a lattice of dimers $\left(H_{x y}=0.07 M_{s}, \omega=12.6 \mathrm{rad} \mathrm{s}^{-1}\right)$. A blue arrow is assigned to each dimer with a green dot as the head following the arrow representation. (d) Random FPL phase $\left(H_{x y}=0.11 M_{s}, \omega=12.6 \mathrm{rad} \mathrm{s}^{-1}\right)$; one small loop is highlighted in red. (e) Order parameters $|\boldsymbol{M}|$ and $\phi_{N}$ versus time for (c). Inset: schematic showing the definition of $\phi_{N}$, as the fraction of vertices having one incoming and one outgoing arrow. (f) Diagram in the $\left(\omega, H_{x y}\right)$ plane illustrating the various colloidal phases. Filled points indicate the locations of (c) and (d).

the stray field. On the surface of the FGF, the $\boldsymbol{H}_{x y}$ field strengthens (or weakens) the stray field above the interstitial region, depending on whether the magnetic field lines are parallel (or antiparallel) to the applied field.

Next, we explore the stationary phases that emerge when a disordered lattice of dimers is subjected to an annealing by varying the amplitude and frequency of $\boldsymbol{H}_{x y}$. In order to characterize the dimer arrangement, we use the arrow representation as originally introduced by Elser and Zeng [46] for the spin- $\frac{1}{2}$ kagome antiferromagnet, shown in Fig. 3(a). Since each dimer sits on one of the three sides of a triangular minimum, it can be uniquely associated an arrow pointing from the dimer center to the free corner of the triangle. Each triangle has three nearest neighbors, one outgoing arrow and from zero to three incoming ones. In the latter case, two arrows from adjacent triangles can superimpose and create a defect. FPLs occur when there are no such defects and the arrows form closed loops that visit each lattice vertex only once. Two representative images 
illustrating a long range striped phase and a random FPL state obtained with this mapping are shown in Figs. 3(c) and 3 (d), where to guide the eye the arrows have a green dot as the head. The first state is characterized by parallel stripes of arrows with a mean director given by the orientation of the applied field, which breaks the symmetry of the underlying potential. Sliding symmetries characterized by parallel stripes having opposite directions, as observed in Ref. [30], are not possible here. A typical labyrinth and a disordered phase are showed in Ref. [31]. To distinguish between the various phases, we measure two order parameters: a Néel type parameter $|\boldsymbol{M}|$ [47], and the fraction $\phi_{N}$ of vertices in the lattice having exactly one incoming and one outgoing arrow, as defined in the schematic of Fig. 3(e). The nematic ordering is characterized by high values of both order parameters. Random FPLs have lower values of $|\boldsymbol{M}|$ since the stripes break into smaller loops, but conserve a high fraction in $\phi_{N}$, Fig. 3(b). In contrast, disordered states are characterized by low values of both parameters. Moreover, from the diagram shown in Fig. 3(f), it emerges that the transition between these phases depends weakly on $\omega$ and mainly on $H_{x y}$, which controls the interaction strength. The stripe ordering is observed for $H_{x y} \lesssim 0.8 M_{s}$. Increasing $H_{x y}$, the dimers interact strongly and the stripes start to break up, forming an intermediate labyrinthlike pattern (Fig. S3 in Ref. [31]). The bending of the stripes at high values of $H_{x y}$ can be understood by considering the effect of the time-averaged dipolar interactions [48]. In the absence of a FGF, these interactions are attractive and force a chain of particles like pairs of dimers to aggregate into a compact cluster [49]. The presence of the honeycomb lattice prevents the formation of these clusters, but the stripes can more easily break due to the loss of the synchronization of some composing dimer. For $H_{x y} \sim 0.11 M_{s}$, the strings of arrows completely break into smaller FPLs randomly distributed above the film. In particular, in many FPL states found, we observe a large fraction of elementary loops formed by six touching arrows, which can have both senses of rotations, Fig. 3(d). For fields larger than $H_{x y} \gtrsim 0.12 M_{s}$, these interactions are so strong that they induce complete melting of the lattice.

Finally, we explored the melting process in the dimer lattice, which can be induced by either decreasing $H_{z}$ or, as commented previously, by further increasing $H_{x y}$ such that dipolar interactions completely dominate. The latter case is illustrated in Figs. 4(a)-4(c), and it features a two stage melting transition. For $H_{x y}<0.03 M_{s}$, the applied field is unable to completely rotate the dimers, and the system remains frozen in the initial ordered state, Fig. 4(a). Increasing $H_{x y}$ induces a transition towards a partially ordered state, when the dimers perform localized rotational motion, but the system keeps its positional order, Fig. 4(b). Finally, for $H_{x y} \gtrsim 0.12 M_{s}$ a second disorder transition occurs when the dimers break up and reform, exchanging particles as the field is rotating, MovieS4 in Ref. [31]. The system forms a liquid phase where strong attractive dipolar
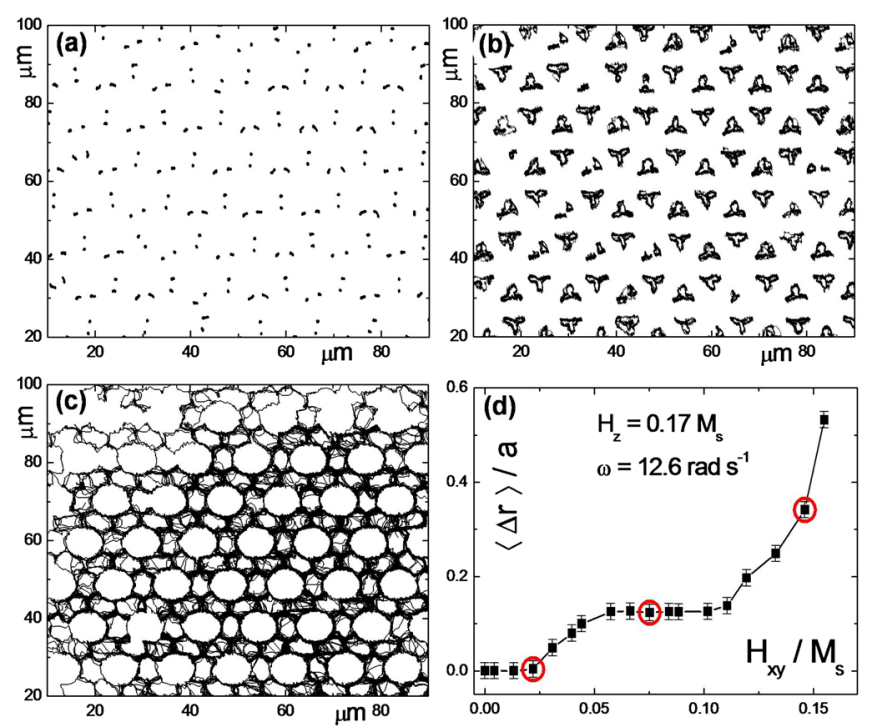

FIG. 4. (a)-(c) Colloidal trajectories (lines) for dimers subjected to an external precessing field with angular frequency $\omega=$ $12.6 \mathrm{rad} \mathrm{s}^{-1}$ and amplitudes $H_{z}=0.17 M_{s}$ and $H_{x y}=0.02 M_{s}$ for (a) MovieS2 in Ref. [31], $H_{x y}=0.07 M_{s}$ for (b) MovieS3 in Ref. [31], and $H_{x y}=0.14 M_{s}$ for (c) MovieS4 in Ref. [31]. (d) Average displacement $\langle\Delta r\rangle$ versus amplitude of the in-plane rotating field $H_{x y}$. Gray (red) circles denote the location of (a)-(c).

forces favor the exchange of particles between nearest interstitials when two dimers rotate close to each other. To quantify this two-step transition, the globally averaged particle displacement $\langle\Delta r\rangle$ is measured for each applied field. We find that both transitions are rather smooth, second-order-like, and characterized by the presence of a finite step of the order parameter in the intermediate phase, Fig. 4(d). A similar melting scenario with finite steps in $\langle\Delta r\rangle$ has been previously predicted via numerical simulations [16]. However, melting here is induced by increasing the dipolar coupling between the particles, rather than decreasing the substrate strength in favor of thermal fluctuations $[13,14]$.

In summary, we realize a frustrated colloidal molecular crystal composed of self-assembled microscopic dimers interacting above a magnetic honeycomb lattice. The system reveals a rich phase behavior when dimer-dimer interactions compete with substrate strength. These interactions can be tuned in situ via application of a rotating field. The dimer covering of periodic lattices can be mapped to Ising systems [50,51], or can be used as a simplified model for the adsorption of diatomic molecules onto a surface, like $\mathrm{N}_{2}$ on graphite [52], lattice gas systems [53], and tiling problems [52]. Yet, the transport properties of bound dimers on a periodic lattice [54], such as DNA linked colloidal doublets [55], is also an interesting future avenue that can be explored with the presented system.

I acknowledge T. M. Fischer for stimulating discussions and Tom H. Johansen for the FGF. This work was 
supported by the European Research Council via Project No. 335040 and by the "Ramon y Cajal" program (No. RYC-2011-07605).

*ptierno@ub.edu

[1] R. Moessner and A. P. Ramirez, Phys. Today 59, No. 7, 24 (2006).

[2] G. H. Wannier, Phys. Rev. 79, 357 (1950).

[3] G. F. Newell and E. W. Montroll, Rev. Mod. Phys. 25, 353 (1953).

[4] H. Shin, K R. Purdy Drew, J. R. Bartles, G. C. L. Wong, and G. M. Grason, Phys. Rev. Lett. 103, 238102 (2009).

[5] M. Nixon, E. Ronen, A. A. Friesem, and N. Davidson, Phys. Rev. Lett. 110, 184102 (2013).

[6] S. N. Dorogovtsev, A. V. Goltsev, and J. F. F. Mendes, Rev. Mod. Phys. 80, 1275 (2008).

[7] S. M. Giampaolo, G. Gualdi, A. Monras, and F. Illuminati, Phys. Rev. Lett. 107, 260602 (2011).

[8] Y.-Z. You, Z. Chen, X.-Q. Sun, and H. Zhai, Phys. Rev. Lett. 109, 265302 (2012).

[9] M. Seo, H. K. Choi, S.-Y. Lee, N. Kim, Y. Chung, H.-S. Sim, V. Umansky, and D. Mahalu, Phys. Rev. Lett. 110, 046803 (2013).

[10] Y. Han, Y. Shokef, A. M. Alsayed, P. Yunker, T. C. Lubensky, and A. G. Yodh, Nature (London) 456, 898 (2008).

[11] R. F. Wang, C. Nisoli, R. S. Freitas, J. Li, W. McConville, B. J. Cooley, M. S. Lund, N. Samarth, C. Leighton, V. H. Crespi, and P. Schiffer, Nature (London) 439, 303 (2006).

[12] C. Nisoli, R. Moessner, and P. Schiffer, Rev. Mod. Phys. 85, 1473 (2013).

[13] M. Brunner and C. Bechinger, Phys. Rev. Lett. 88, 248302 (2002).

[14] C. Reichhardt and C. J. Olson, Phys. Rev. Lett. 88, 248301 (2002).

[15] R. Agra, F. van Wijland, and E. Trizac, Phys. Rev. Lett. 93, 018304 (2004).

[16] C. Reichhardt and C. J. Olson Reichhardt, Phys. Rev. E 71, 062403 (2005).

[17] A.Šarlah, T. Franosch, and E. Frey, Phys. Rev. Lett. 95, 088302 (2005).

[18] S. El Shawish, J. Dobnikar, and E. Trizac, Soft Matter 4, 1491 (2008).

[19] R. I. Baxter, Exactly Solved Models in Statistical Mechanics (Academic Press, New York, 1982).

[20] F. Alet, J. L. Jacobsen, G. Misguich, V. Pasquier, F. Mila, and M. Troyer, Phys. Rev. Lett. 94, 235702 (2005).

[21] A. W. Sandvik and R. Moessner, Phys. Rev. B 73, 144504 (2006).

[22] K. W. Wojciechowski, D. Frenkel, and A. C. Branka, Phys. Rev. Lett. 66, 3168 (1991).

[23] M. F. T. R D. Diehl and J. S C. Fain, Phys. Rev. Lett. 48, 177 (1982).

[24] H. W. J. Blöte and B. Nienhuis, Phys. Rev. Lett. 72, 1372 (1994).

[25] B. Duplantier, Phys. Rev. Lett. 81, 5489 (1998).

[26] K. O'Holleran, M. R. Dennis, F. Flossman, and M. J. Padgett, Phys. Rev. Lett. 100, 053902 (2008).

[27] A. Nahum, J. T. Chalker, P. Serna, M. Ortuno, and A. M. Somoza, Phys. Rev. Lett. 107, 110601 (2011).
[28] L. D. C. Jaubert, M. Haque, and R. Moessner, Phys. Rev. Lett. 107, 177202 (2011).

[29] J. L. Jacobsen and F. Alet, Phys. Rev. Lett. 102, 145702 (2009).

[30] G.-W. Chern, C. Reichhardt, and C. J. Olson Reichhardt, Phys. Rev. E 87, 062305 (2013).

[31] See Supplemental Material at http://link.aps.org/ supplemental/10.1103/PhysRevLett.116.038303, which includes Refs. [32-42], more experimental and theoretical details and four video clips illustrating the particle dynamics.

[32] G. Misguich, D. Serban, and V. Pasquier, Phys. Rev. Lett. 89, 137202 (2002).

[33] P. Tierno, F. Sagués, T. H. Johansen, and T. M. Fischer, Phys. Chem. Chem. Phys. 11, 9615 (2009).

[34] P. Tierno, Phys. Rev. Lett. 109, 198304 (2012).

[35] P. Tierno, Langmuir 30, 7670 (2014).

[36] P. Tierno, T. H. Johansen, and T. M. Fischer, Phys. Rev. Lett. 99, 038303 (2007).

[37] W. F. Druyvesteyn, D. L. A. Tjaden, and J. W. F. Dorleijn, Philips Res. Rep. 27, 7 (1972).

[38] P. Tierno and T. M. Fischer, Phys. Rev. Lett. 112, 048302 (2014).

[39] A. Soba, P. Tierno, T. M. Fischer, and F. Saguès, Phys. Rev. E 77, 060401 (2008).

[40] P. Tierno, F. Sagués, T. H. Johansen, and I. M. Sokolov, Phys. Rev. Lett. 109, 070601 (2012).

[41] T. H. Johansen, A. V. Pan, and Y. M. Galperin, Phys. Rev. B 87, 060402 (2013).

[42] C. Kooy and V. Enz, Philips Res. Rep. 15, 7 (1960).

[43] On a striped FGF the effective translational diffusion coefficient was measured as $D=3.2 \times 10^{-4} \mu \mathrm{m}^{2} \mathrm{~s}^{-1}$ [44].

[44] P. Tierno, P. Reimann, T. H. Johansen, and F. Sagués, Phys. Rev. Lett. 105, 230602 (2010).

[45] R. Adler, Proc. IRE 34, 351 (1946).

[46] V. Elser and C. Zeng, Phys. Rev. B 48, 13647 (1993).

[47] In analogy with Ref. [30], from the position of the particles in the dimers and from the corresponding position of the nearest magnetic bubble, we calculate the Néel parameter as $\boldsymbol{M}=2 /(\sqrt{3} N) \sum_{i}(-1)^{i} \hat{\boldsymbol{e}}_{i} \quad$ with $\quad \hat{\boldsymbol{e}}_{1} \equiv(0,1)$ and $\hat{\boldsymbol{e}}_{2,3} \equiv$ $( \pm \sqrt{3} / 2,-1 / 2)$ being the three lattice vectors; $(-1)^{i}$ is $+1(-1)$ for an up (down) triangle.

[48] The normalized pair potential between two induced dipoles $\boldsymbol{m}_{i}, \boldsymbol{m}_{j}$ separated by a distance $\boldsymbol{r}_{i j}=\boldsymbol{r}_{i}-\boldsymbol{r}_{j}$ can be written as $U_{m}=\left(\mu_{m} / 4 \pi\right)\left[\left(\boldsymbol{m}_{i} \cdot \boldsymbol{m}_{j}\right) / r_{i j}^{3}-3\left(\boldsymbol{m}_{i} \cdot \boldsymbol{r}_{i j}\right)\left(\boldsymbol{m}_{j} \cdot \boldsymbol{r}_{i j}\right) / r_{i j}^{5}\right]$, with $\mu_{m}$ being the magnetic susceptibility of the medium. Performing a time average of the dipolar energy between two colloids when subjected to a rotating field $\boldsymbol{H}_{x y}$ in the $(x, y)$ plane gives an effective attractive potential in this plane, $\left\langle U_{m}\right\rangle=\left[-\mu_{m} m^{2} / 8 \pi(x+y)^{3}\right]$.

[49] N. Casic, S. Schreiber, P. Tierno, W. Zimmermann, and T. M. Fischer, Europhys. Lett. 90, 58001 (2010).

[50] M. E. Fisher, Phys. Rev. 124, 1664 (1961).

[51] P. Kasteleyn, J. Math. Phys. 4, 287 (1963).

[52] O. G. Mouritsen and A. J. Berlinsky, Phys. Rev. Lett. 48, 181 (1982).

[53] M. Lohöfer, L. Bonnes, and S. Wessel, Phys. Rev. E 88, 052135 (2013).

[54] S. von Gehlen, M. Evstigneev, and P. Reimann, Phys. Rev. E 79, 031114 (2009).

[55] P. Tierno, R. Golestanian, I. Pagonabarraga, and F. Sagués, Phys. Rev. Lett. 101, 218304 (2008). 